\title{
A USER-CENTRED DESIGN FRAMEWORK FOR THE DEVELOPMENT OF SPEECH AND LANGUAGE THERAPEUTIC TOYS
}

\author{
Balzan, Emanuel; \\ Farrugia, Philip; \\ Casha, Owen \\ University of Malta
}

\begin{abstract}
Therapy in early childhood is facilitated through toys and play. While mainstream toys are designed for children, therapeutic toys need to satisfy requirements from clinicians, caregivers, and children. The study presented in this paper investigated the challenges that 22 international toy designers encounter during the design process and whether support is required when developing products for speech and language therapy, through a mixed-method approach. Results show that considerable challenges are encountered during the early design stages. Nonetheless, the toy design process remains unsupported, while no support is available for designers to consider therapeutic needs. Based on the feedback received, eleven requirements were identified upon which a user-centred design support framework was proposed to assist toy designers during the task clarification stage, taking into account the affordances that therapeutic toys should have without inhibiting the creative process.
\end{abstract}

Keywords: User centred design, Early design phases, New product development, Therapeutic toys design, affordances

\section{Contact:}

Balzan, Emanuel

University of Malta

Dept. of Indus. and Manuf. Engineering

Malta

emanuel.balzan@um.edu.mt 


\section{INTRODUCTION}

The motivation of this research concerns the design of therapeutic devices, or as widely known within the medical field, therapeutic toys (TT). Toys in institutional healthcare have been in use for more than five decades (Da Silva et al., 2016). One such application is in speech and language therapy (SALT). Signs of both expressive and receptive speech and language (SAL) difficulties in children can be diagnosed from an early age. Speech and Language Pathologists (SLPs) are clinicians qualified to provide SALT to children from as little as two-years-old. Therapy in early childhood is facilitated through toys and play. Having the right tools is important to support both the clinicians' therapeutic effort in delivering effective intervention and to provide ways to engage children and drive them to work towards the therapeutic goals (Fikar, Güldenpfennig and Ganhör, 2018). In a time where customer demands are becoming more personal, development cycles are shorter and market competition continues to increase, a successful product is determined by how well the customer overall requirements have been satisfied. The study presented in this paper is aimed at investigating the challenges that toy designers find during the design process and whether support is required when creating products for SALT.

The rest of the paper is divided as follows. The next subsections describe what TTs are and outline the methodology of the study. Related works are reviewed in section 2, together with the identified research gap. Section 3 describes a mixed-method study used to understand the designer's reality and identify the requirements for a design framework. The developed framework is explained in section 4 . The results are discussed in section 5 whilst future work and conclusions are drawn in section 6 .

\subsection{Difference between Mainstream Toys and Therapeutic Toys}

Since the aspect of play is central in understanding the development of children, early intervention specialists such as SLPs have learned to adapt mainstream toys (MTs) to their objectives of intervention (Williams and Matesi, 1988). Unlike other consumer products, MTs are designed for children with the purpose of engaging them in play activities but are purchased by adults. In the case of TTs, the main objectives concern the needs of the clinician and the target goals that are set for the child. For therapeutic activities to be effective, clinicians insist that therapy is carried over from the clinic to the home of their clients and performed daily (Cooper, Moodley and Reynell, 1979). Thus, parents, teachers, or any other caregiver have an important role in facilitating therapy outside the clinical environment, causing their requirements to become therapy oriented (Blanco et al., 2016). Additionally, designers need to foresee situations in which the synthesised solution will (and may) be used to create the optimum user experience. From focus groups carried out prior to this study, it was found that SLPs do not have purposefully designed speech and language therapeutic toys (SALTTs) but tend to adapt multiple MTs or create low-tech resources to carry out intervention. This problem is more pronounced in countries where clients need to travel long distance and/or countries that are bilingual, where toys for particular languages do not exist. Given these reasons, a gap in the market exists for SALTTs.

\section{RELATED WORK}

SLPs have fully embraced the use of Information and Communication Technology in assessment, diagnosis and intervention due to the positive impacts it has on both adults and children as indicated by the literature review of (Drigas and Petrova, 2014). The lack of appropriate motivational media leads to a reduction in motivation towards therapy. Furlong et al. (2018) report that by 2017, there were more than five thousands applications (apps) on Google Play and Apple iTunes app stores related to speech and language, but only 132 apps were considered to have therapeutic benefits. Since the 'touch-screen generation' continues to provide highly attractive games, it is unlikely that software solutions can be eliminated. This is also because, software (coupled with the right hardware) provides essentials tools for therapy such as voice recognition, text to speech, video conferencing, and variety of games amongst other. Barr (2019) shows that well-designed media can help children learn, particularly when interacting with other people. In (Rivera et al., 2016; Jadi, 2019), a number of technological elements in smart toys for children with autism were proposed to improve their social communication and interaction. In (Blanco et al., 2016) a framework for the design of assistive products is proposed. Their work is built up 
from a bottom-up approach, describing how assessments do not just evaluate the usefulness of product but also shape the design process when considered from the beginning.

Fikar, Güldenpfennig and Ganhör (2018) propose five generic design lenses for interactive toys to afford therapists, allowing therapists to design play activities. These lenses were developed while observing early intervention specialists during intervention sessions and discussions. The authors state that designers should (i) design features based on the intended reaction from children and their developmental status (ii) embed technology and provide control to limit or enhance them, (iii) design toys that provide rich play experiences, (iv), design toys that offer flexibility and the ability to improvise, and (v) design toys that are practical, and easy to use and maintain. Although these lenses do not provide enough details to develop SALTTs, they provide good principles on how to keep a child motivated during therapy. In these articles, the third group of agents discussed in Section 1.1, the caregivers, were not involved in any of the stages. The argument presented in this paper is that therapeutic toys must afford therapists, children, and parents, or as have been generalised in this paper, clinicians, children and caregivers. In view of this, a thorough understanding of affordances for therapeutic toys is required.

Norman's final view about affordances is that they result from the cognitive understanding of the perceived and actual properties of an object that determines how the object can possibly be used (1999). Maier and Fadel (2009), state that unlike affordances, functions are form independent but they "cannot be fully explained without reference to its physical embodiment", meaning that functions can be characterised as affordances. However, not all functions of a product can be perceived through its design. Maier and Fadel also relate the affordances of an object to the behaviour of a user by considering affordances to be potential uses of an artefact, stating that structures of systems determine affordances and in turn describe the possible behaviours. In the case of a SALTT, it must intentionally afford SAL intervention-ability. Nevertheless, by using adapted MTs in therapy, they unintentionally facilitate SAL intervention through the affordances of play. Although making an object's perceivable affordances highly identifiable is a good design practise, in the case of TTs, affordances related to therapy should remain hidden because objects that are normally prescribed or used in particular settings such as clinics can be stigmatic (Blanco et al., 2016). Norman stated that "in some cases, it is best to hide the real affordance" (1999). The recent work of Davis (2020) explains that artefacts afford differently to different people and with varying intensities. Therefore, certain features of SALTTs should be visible to the SLPs only because they are qualified to assess a child and prepare an adequate intervention programme. On the other hand, the perception of play must be made highly evident to children while diminishing the therapy perception as much as possible. Similarly, one should consider the dexterity of caregivers that would allow them to compliment the work of the SLP at home. Mapping requirements into affordances is necessary to infer the capabilities of the artefact with respect to its user.

\subsection{Methodology}

This paper addresses the following research questions: (i) which stage of the design process do toy designers find most difficult? (ii) do experienced and novice toy designers alike need support in designing smart TTs? and if yes, (iii) what kind of support do designers need? By following the design research methodology by (Blessing and Chakrabarti, 2009), a descriptive study was carried out to observe the current toy designers' reality, to understand how the design process of TTs differs from MTs and to determine the phenomena of the design problem. In parallel, literature on the development of related devices was reviewed to understand the knowledge gap in creating such devices. The results of these two activities will establish the requirements for the support of SALTT design. These findings lead to answering the final research question, which is, (iv) how to develop a user-centred framework that enables designers to consider the clinicians', caregivers' and children's functional and non-functional requirements, towards an artefact, that engages and motivates children towards the therapeutic effort?

\section{IDENTIFYING THE TOY DESIGNERS' NEEDS THROUGH A MIXED METHOD APPROACH}

A study was carried out with 22 stakeholders (12 males and 10 females) whose toy industry experience varied between 1 to 30 years, with a mean of 10.70 years and a standard deviation of 9.58 years. Out of the 22 participants, 17 were toy designers, of which 6 are (co)owners. The other five participants were 
product managers who are directly involved in the toy development process. Eleven participants work in big companies (> 60 employees), nine work with small companies and two are freelancers. It is important to highlight that it was difficult to recruit participants since no contacts were available locally. Only 10 participants agreed to participate in live semi-structured interviews while the rest requested to fill an offline questionnaire. From the feedback received, it was noted that similar results were obtained. The questionnaire was divided into three parts. The first section aimed to capture the current practices of toy design, the second section was about the use of decision support tools during toy design, and the third section was specifically about the design of therapeutic toys. Three of the live interviews were carried out in person after visiting a toy factory cluster in Spain, while the other interviews were conducted via Skype. The interviews, which lasted between 60 to 90 -minutes, were based on the questionnaire. However, with the semi-structured interviews, discussions followed every question to obtain further insight. All interviews were voice recorded and transcribed verbatim for post analysis. Key responses from the participants were coded, sorted and clustered into identified themes by following the procedure of (Braun and Clarke, 2006) for thematic analysis. The identified core themes answer the research questions are described in the following sub-sections. Quantitative input from all the participants was used to infer trends among toy designers. This research protocol and the data collection instruments were reviewed and approved by the ethics committee of the University of Malta.

\subsection{Theme 1: Toy design is mostly not user-centred}

\subsubsection{Acquiring Requirements}

According to the participants, a toy is conceived either (i) from an idea, following observations (23.70\%) or through intuition (8.50\%); (ii) from an identified need, either as a market gap (27.10\%) or by following the competition and market trends $(22.05 \%)$, or (iii) from an external customer request $(18.65 \%)$. Whilst every designer confirmed that the overall toy design process is similar to the methodology highlighted in (Pahl et al., 2007), there were two main differences on the way requirements are collected and passed to the designer. There are toy factories where the requirements are received from major supermarket brands, rather than directly from the end users. In such cases, the requirements are stated in the agreement. On the other hand, when the need for a new toy comes within the company, depending on the size of the organisation, designers may or may not be involved in the requirement collection stage. Designers may be resident employees or freelancers. Most of the times, the requirements are passed either in a short report (50\%) containing an image portraying the vision of the marketing and sales team / management, or verbally (18.20\%) during meetings. Sometimes, designers propose new ideas and requirements are self-established via a team effort (31.8\%).

\subsubsection{End-user involvement}

In large companies, end-users are likely to be involved during prototype testing in the embodiment design stage because "before investing [money], we have to be sure that this product is going to work" as "testing comes from uncertainties within the company". In contrast, small companies, involving entrepreneurs are more likely to go through the design process all by themselves. It has been noted that such companies tend to be closer to the users, and an idea is tested during the multiple design stages. Furthermore, "when you are working on a college project, you apply an accurate design methodology. When you are freelancing, you also apply research. But when you are in the corporate world, some companies do not spend much of their time in research.".

\subsection{Theme 2: Early Design Support}

\subsubsection{Design decisions}

End users may not be involved during the design process because of the experience that designers build up along the years. Experience allows them to take decisions: "decisions in toys are often gut feelings because you believe from experience this idea will work. Sure, there are inputs at concept stage to help understand what is popular but as the process develops, then cost normally drives most decisions". Designers were asked to rank the design stages based on the most important decisions that are taken. Their feedback was weighted using the Rank Order Centroid (ROC) method (Sureeyatanapas, 2016). The result, which is illustrated in Figure 1(a), shows that the critical decisions are taken at task clarification stage, followed by the concept, embodiment, and final design stages. 


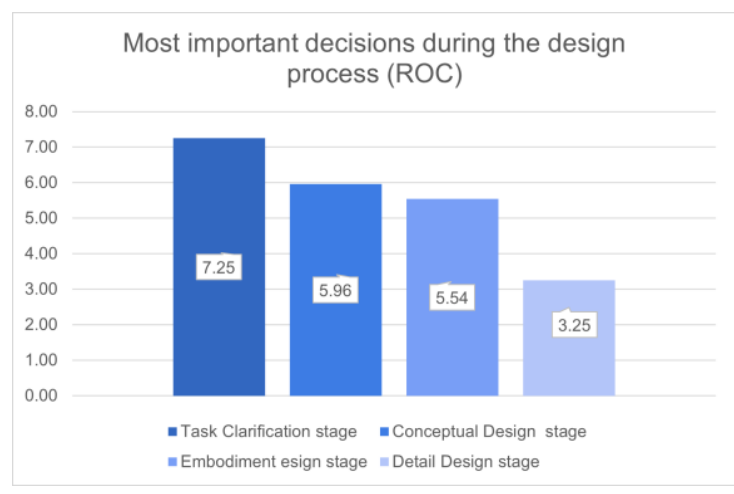

(a)

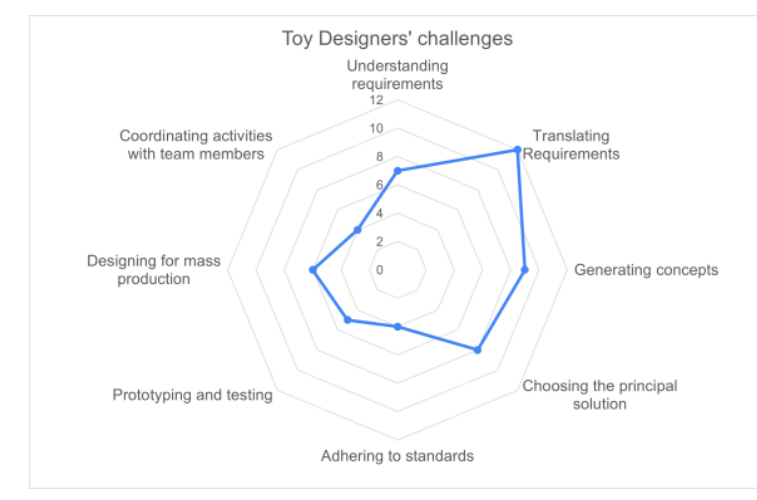

(b)

Figure 1: Toy designers' most important (a) decisions and (b) challenges

All participants agreed that experience and creativity are the most important assets for a toy designer. A challenging activity is "defined by what experience you have". However, when asked to rate how difficult they find it to satisfy all customer requirements, $23 \%$ claimed that accommodating requirements is "Very Hard", $41 \%$ responded "Hard", and 36\% said "Medium". Although this is a subjective indication, a Kruskal Wallis test on the degree of challenge in satisfying customer requirements vis-à-vis the years of experience of the designer showed, that there is no significant difference $(p$-value $=0.858)$. This means that similarly experienced designers think the same way.

\subsubsection{Designer's challenges}

The designers were asked to mention which activities they find most difficult. As shown in Figure 1(b), their challenges reside in the transition between the task clarification to the concept design stage, starting from understanding of the requirements to translating the requirements into initial concepts. The effect of these challenges propagates into the concept stage as many designers find it difficult to choose the principal solution. Understanding the requirements is difficult because "some of the requirements are not necessarily black and white - there's lots of grey space", and "translating requirements is not a formula. Even with experience, this task remains difficult because it requires thinking and creativity." and "you can only interact with so many end users. And so, you only have the perspective of those end users in your biases". Designers stated that "if we understand correctly the requirements of the product, we can make the right product to the market and consumer". The toy designers confirmed that unless the toy is commissioned by a customer, the product specifications are not available until prototyping has started. In fact, "when you get to that first prototype, you're actually making a hypothesis that this is the best approach based on everything you know". Although experience and tacit knowledge are important characteristics of the designer, all participants said that they refer to past and competitors' toys which would be in special rooms or labs. However, when referring to old toys, care must be taken due to frequent changes in safety standards.

\subsubsection{Design Support tools for MT}

All participants confirmed that no design support tools are used to support decision making, except for one product manager who mentioned the use of spreadsheets to check whether the investments involved would still make the product profitable. Six designers referred to 3D-CAD software that they use in designing products. A designer referred to the challenges in interpreting design guidelines and the fact that they do not provide "feedback on whether or not we've executed the principle correctly".

When asked if a support tool would help in designing better toys, $27.27 \%$ of the participants strongly agreed while $36.36 \%$ agreed. $9.09 \%$ of the participants disagree and strongly disagree that a support tool would help. A novice designer believes "that [a tool] would be quite useful to justify your decisions based on real data. So, having massive amount of data of people's preferences you can make your decision". Only $18.18 \%$ of the participants provided a neutral reply and this was mainly because they could not understand how a design support tool could assist them. The Kruskal Wallis test $(\mathrm{p}$-value $=0.349)$ reveal no significant difference between their reply and the years of experience, meaning that similarly experienced designers think it the same way. The participants were also asked whether the increased presence of end-users during the design process would help to design better 
toys, $40.91 \%$ strongly agreed and $36.36 \%$ agreed, whereas $13.64 \%$ gave a neutral feedback and $9.09 \%$ disagreed. Again, no significant difference was found $(\mathrm{p}$-value $=0.739$ ).

Participants were asked to rate the most important design factors for MT. The ROC weighting method revealed that the primary considerations are safety, function and play value. This cluster of design factors was followed by target skills and cost of manufacturing. The third cluster of design factors consist of aesthetics, durability, ergonomics, and ease of manufacturing. This result shows that aesthetical factors are less important than functional, play value and target skill factors in toys because "if it's not very nice, children would still find it interesting. It is OK for them. What is more important is that children can learn something with it". Some argued that the aesthetic is "the first thing to sell in a product", but related to that there are "cost of manufacturing and ease of manufacturing... I can have a very nice product, it meets the target, everything is safe, it is easy to use. But if the price is not the one that the [end user] is OK to pay, then I don't have a product because at the end I have to sell the product".

\subsubsection{Design Support tools for therapeutic toys}

$54.5 \%$ of the participants strongly believed that the design process for TTs and MTs is the same. On the other hand, $9.1 \%$ and $13.6 \%$ of the participants strongly disagreed and disagreed respectively, claiming that "the process of designing is very similar, apart from the identification of the needs". $22.8 \%$ of the participants gave a neutral reply. A Kruskal Wallis test shows no significant difference between their reply and their years of experience (p-value $=0.566)$. Most of the participants $(86.4 \%)$ strongly believe that identifying the requirements of TTs is more difficult than for MT, while only $13.6 \%$ disagreed. The Kruskal Wallis test showed that there is no significant difference ( $\mathrm{p}$-value $=0.068$ ). This small $\mathrm{p}$-value resulted because out of the 3 participants that disagreed, 2 participants have over 20 years of experience and the other participant has only 4 years of experience.

Three similar consecutive questions were asked to understand whether a design support tool would be suitable just for MTs, just for TTs or for both. Their responses changed from $9.1 \%$ to $13.6 \%$ to $59.1 \%$ strongly agree, respectively. In the latter case, $9.1 \%$ said that they disagree while $31.8 \%$ gave a neutral reply. A significant difference ( $\mathrm{p}$-value $=0.047$ ) was found when a Kruskal Wallis test was carried out, showing that the years of experience affect the need for support tools. On a closer look at the data the participants with over 20 years of experience gave varied answers: two disagreed, two strongly agreed and two gave a neutral feedback on whether a support tool would be useful. Most participants with less than 20 years of experience strongly agreed the idea of having a support tool for both MT and TT.

Finally, when asked at which stage support would be needed to assist TT design, $72.7 \%$ of the participants said during task clarification, $18.2 \%$ said during concept design and $9.1 \%$ said during the detail design. The two participants who preferred support in the last stage of the process also stated that their biggest challenge is designing for mass production. No significant difference was found $(p$-value $=0.38)$ when testing for difference across replies with respect to their years of experience.

\subsection{Theme 3: SALTT Framework requirements}

From this study, eleven requirements for a TT design support framework emerged. These are:

R1: Support during task clarification stage - Most designers realised that support is needed before starting the conceptual stage because "the task clarification has to be done well and one must know about the specific target".

R2: Provide therapy-related knowledge from reliable sources - Therapy related requirements need to be available from the beginning to help designers design appropriate TTs. All participants strongly agreed that when designing TT designers should consult with clinicians.

R3: Not hinder the creative process - Participants voiced that the tool should only provide guidance and the design process should remain creative.

R4: Consider checklists and user personae for requirements - Some participants suggested that the format of the design support should be like " a checklist. Like, does it have all these features? Does it meet all these things? Is a child going to be able to navigate through it? Are things picture based 
rather than word based? Are the words assessed at the level of the child whose playing it?". Personae help in understanding the users' demands and wishes by empathising with their needs. This includes understanding of the different contexts of use, their abilities, development level, cultural factors, etc. The framework should "understand the age parameters, the most important thing for a person of each age, their gender" and be very granular so you can select information for a specific customer group".

R5: Provide access to users' preferences, market trends, past products, and manufacturing knowledge The framework should "give you an idea of the preferences and needs of target audience" by keeping the users "part of the equation"; include reference libraries with past toy products knowledge such that the designers are able to reference them or get inspired; capture how existent toys or certain features of toys are used during therapy to inspire designers how to address therapeutic requirements; understand market trends and gaps such that it "guarantees a million sales"; and include material and production knowledge libraries which would allow them to refer to when translating customer requirements.

R6: Understand design consequences - As stated in the second theme, the task clarification stage is critical in defining the path that the final design will take. Such tool can provide the "research for inspiration to find technical solutions. Maybe also to help me understand the cost of different solutions".

R7: Support in providing richer experiences - Therapy and play should be fun to get the most benefits. In fact, function and play value were rated important attributes of toys. TTs should be more than just tools but afford characteristics which allow children to engage in therapy.

R8: Consider safety standards - "Standards are very important for the safety of children... they affect the design and the price". Standards determine whether toys may be launched into the market or not.

R9: Have a computer-based design tool. The majority (81.8\%) of the participants said that a design support should be computer-based. It was noted that in some toy factories, tasks are still carried out on paper - "the way we work here is more paper-based. If you are thinking about the future, I would say as a computer-based tool because having paper it is always a mess".

R10: Be updateable - The participants said that "it only make sense to use such tool if the information is up-to-date" since "the market is very dynamic and so it needs to be updated regularly". Also, "standards are changing every year. We have a lot of old products that are not suitable for today's market".

R11: Provide economic and production feasibility insight - Designers are required to stay within target budgets even at early design stages because "it is easy to develop 100's of ideas, but the art to picking the winners and the ones with most overall commercial viability" determines if that idea can be turned into a product or not. The trick is "balancing play features with target cost given the importance of certain play features in success of past products".

\section{THE D-SALTT USER-CENTRED DESIGN FRAMEWORK}

Based on the identified requirements and the need to consider TT's affordances, a user-centred design framework for the Design of Speech And Language Therapeutic Toys (D-SALTT) is being proposed and illustrated in Figure 2. The framework is being proposed for both novice and experienced toy designers stepping in the field of speech and language therapeutic devices. The framework consists of five layers. At the core, there is the Stakeholders Layer (RI) consisting of the end-users and product development stakeholders whose requirements go directly to the designer. A computer-based tool $(R 9)$ needs a user interface such that the designer can handle all the requirements easily. The second layer which is the User Interface Layer allows the designer to go through the tasks of the task clarification stage.

These tasks are shown as frames. A Requirements Generation Frame (1) allows the designer to input the provisional requirements into the tool $(R 1)$ and the Checklist, Personae and Scenarios Consideration Frame (2), presents the user with a checklist, use-case scenarios and/or different personae that can be considered as part of the initial requirements (or constraints) identification $(R 4)$. 


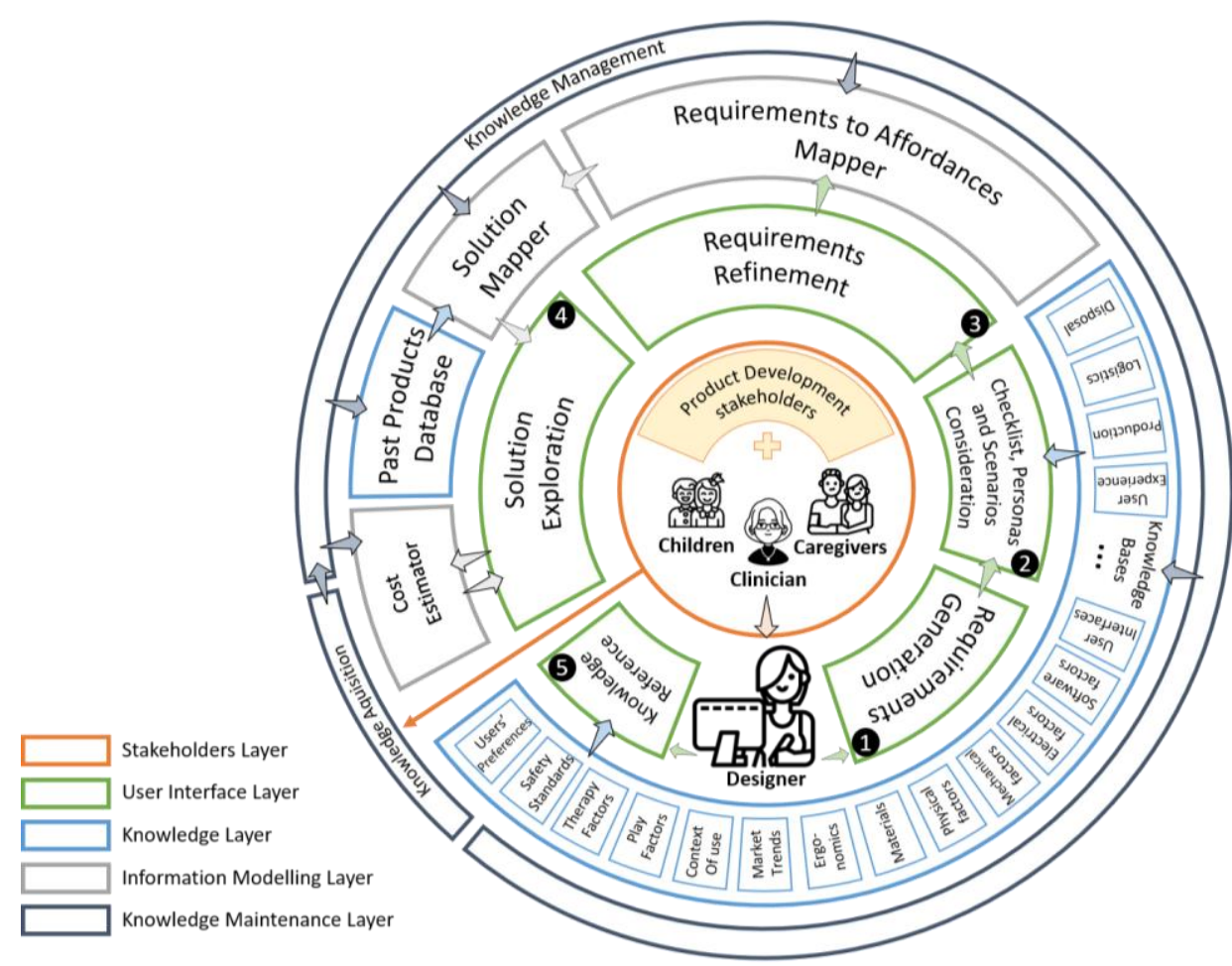

Figure 2: D-SALTT user-centred design framework

The Requirements Refinement Frame (3) is where requirements are extended if quantitative information is known. The Solutions Exploration Frame (R4) provides inspiration to the designer based on the requirements $(R 2, R 3)$. In the Knowledge Reference Frame (5), the designer can access the knowledge bases (libraries) at any time for reference $(R 5, R 8)$. This frame and the Checklist, Personae and Scenarios Consideration Frame connect with the Knowledge Layer which contains several knowledge facets and a database on existing MT utilised in therapy (R5). The Requirements Refinement Frame pushes the requirements to the Information Modelling Layer, where the Requirements to Affordances Mapper Frame $(R 7)$ converts them into a predefined format based on the theory of affordances as explained below. In this way, the Solution Mapper Frame can retrieve related past products examples to the Solution Exploration Frame $(R 5, R 6)$ as input for the next stage of design, i.e., concept design stage. Since the participants expressed concerns on the economic feasibility of ideas, a Cost Estimator Frame (R11) in the Information Modelling Layer is being proposed. Note that the purpose of this framework is not to try and find all possible requirements from the beginning. Pahl et al. state that any attempt to do this "will fail and would cause considerable delays" (2007). Since user needs and preferences, knowledge and models change over time, a Knowledge Maintenance Layer (R10) has been added to the framework where new knowledge is passed to the Knowledge Acquisition Frame and then handled by the Knowledge Management Frame which updates the respective libraries and models.

Maier and Fadel (2009) describe that the design of artefacts should be based on relational theory where affordances are used "to describe the relationships between designers, artefacts and users in design". It is the affordances created by the designer which determine how users should use or interact with artefacts, and so one should avoid unintended negative affordances such as stigmatism. The work of (Kudrowitz and Wallace, 2010) has been fundamental in looking at toys as objects that afford play. Mertala et al. (2016) state that toys have the functional manipulation potential through their attractive, pragmatic, and adaptive affordances. However, the difference of a TT from a MT lies in the therapeutic potential that the toy will have. The therapeutic potential is defined by how the artefact can satisfy speech and language assessment and intervention needs by considering language development in children. Therefore, the Requirements to Affordances Mapper Frame shall link the desired requirements into affordances that combine the therapeutic and play needs in the light of the environment and context the TT will be used in, and any other impairments the user may have to maximise accessibility of the TT. 


\section{DIscussion}

This study has showed how the current toy design process is unsupported even though there are many challenges. In fact, toy designers struggle to start conceptualisation because no guidance is available for them to understand the requirements. As a result, the realised artefact may be different than the requirements. Through this study, it was possible to answer the research questions stated in the beginning. The series of questions led to a mixed method approach where the qualitative feedback was confirmed quantitatively and findings from literature. The first question identified that designers need support in the task clarification. Pahl et al. (2007) mention that this stage is challenging as not all requirements are explicitly stated. The second question examined for whom such tool would be suitable.

Overall, the idea of having a support tool was welcomed by both experienced and novice toy designers, even though some of them could not understand how it could support them. These doubts could have risen because the study was of an exploratory nature, meaning that it was not yet known where support would be given and how. Nonetheless, the questions were structured in a way that their feedback characterised the support needed. In response to the third research question, the participants claimed that clinical input would be required when designing TTs. Literature shows that caregivers' requirements should also be considered. Designers also explained that constraints from budget and manufacturing are considered as input requirements. To answer the third research question, the framework was equipped with a non-exhaustive list of multidisciplinary Knowledge Bases that designers can reference during the design. The framework was realised based on the 11 requirements that emerged from the study, whereas findings from literature contributed to define the need for a Requirements to Affordances Mapper Frame. The end-users were placed in the core of the framework so that the functional and non-functional demands and wishes can be captured by the designer. Thus, answering the fourth research questions. Although this framework considers only the task clarification stage, the framework can be adapted so that all design stages revolve around the end users. This brings a shift from a designer-centric approach towards a user-centric approach, promoting participatory design, where designers and end-users can co-design therapeutic solutions.

Note that the overall aim of this framework is to help designers acquire the requirements for speech and language therapeutic toys, that is, the needs of the prospective end users (SLPs, parents and children) whilst showing examples how such requirements were implemented in mainstream or adapted toys. In this way, designers can explore the possibilities and understand better such requirements, without imposing solutions and affect their creativity. In (Darlington and Culley, 2002), requirement elicitation tools have been reviewed and categorised as descriptive or prescriptive support tools, whereas in (Berkovich, Leimeister and Krcmar, 2011) the necessities for requirements engineering (RE) tools for product service systems are detailed. Such literature continues to emerge in the field of RE because of the importance in trying to 'get the design right the first time'. The Quality Function Deployment (QFD) methodology has been integrated with RE tools to support designers prioritise requirements after they are obtained. Although the QFD methodology can be integrated with the presented framework, requirements prioritisation is beyond the scope of the current work. On the hand, the problem with generic models for requirements elicitation is that they do not cater for the specific need of different users in particular use contexts. By focusing on all the users' needs and the usage scenarios, the framework provides user-centric design support.

Limitations of this study are related to the small sample size of 22 international participants, where 12 of them responded to the questionnaire online by providing both quantitative and qualitative feedback. Considering that half of the participants had between 10 - 30 years of experience allowed the collection of reliable responses, which most of the time agreed with those of less experienced participants. Furthermore, no inter-rater reliability was performed on the thematic analysis, but the three highlighted themes were backed with quantitative data.

\section{CONCLUSION AND FUTURE WORK}

This contribution has put forward arguments that toy designers need support during the task clarification stage to better understand and translate the customer requirements. From a mixed study involving 22 international toy designers, 11 requirements were identified upon which a user-centred design support framework was proposed to assist toy designers during task clarification to understand the requirements 
of clinicians, caregivers and children for TT without inhibiting the creative process. As future work, the D-SALTT framework will be evaluated by toy designers. Along with other user studies that were carried out, a computer-based support tool will be developed and used with respect to a SALT case study. The tool will be showcased to toy designers and their feedback will be used to improve it further.

\section{ACKNOWLEDGMENTS}

This work was supported by the Malta Council for Science and Technology (MCST), through the FUSION Technology Development Programme 2016 (R\&I-2015-042-T). The authors would like to thank the toy designers who participated in this study.

\section{REFERENCES}

Barr, R. (2019) 'Growing Up in the Digital Age: Early Learning and Family Media Ecology', Current Directions in Psychological Science, 28(4), pp. 341-346. https://dx.doi.org/10.1177/0963721419838245.

Berkovich, M., Leimeister, J. M. and Krcmar, H. (2011) 'Requirements engineering for product service systems: A state of the art analysis', Business and Information Systems Engineering, 3(6), pp. 369-380. https://dx.doi.org/10.1007/s12599-011-0192-2.

Blanco, T. et al. (2016) 'Xassess: crossdisciplinary framework in user-centred design of assistive products', Journal of Engineering Design, 27(9), pp. 636-664. https://dx.doi.org/10.1080/09544828.2016.1200717.

Blessing, L. T. M. and Chakrabarti, A. (2009) 'DRM, a Design Research Methodology', p. 397. https://dx.doi.org/10.1007/978-1-84882-587-1.

Braun, V. and Clarke, V. (2006) 'Using thematic analysis in psychology', Qualitative Research in Psychology, 3(2), pp. 77-101. https://dx.doi.org/10.1191/1478088706qp063oa.

Cooper, J., Moodley, M. and Reynell, J. (1979) 'The Developmental Language Programme. Results from a Five Year Study', International Journal of Language \& Communication Disorders, 14(1), pp. 57-69. https://dx.doi.org/10.3109/13682827909011346.

Darlington, M. J. and Culley, S. J. (2002) 'Current research in the engineering design requirement', Proceedings of the Institution of Mechanical Engineers, Part B: Journal of Engineering Manufacture, 216(3), pp. 375-388. https://dx.doi.org/10.1243/0954405021520049.

Davis, J. L. (2020) How Artifacts Afford: The Power and Politics of Everyday Things. The MIT Press (Design Thinking, Design Theory). https://dx.doi.org/10.7551/mitpress/11967.001.0001.

Drigas, A. and Petrova, A. (2014) 'ICTs in Speech and Language Therapy', International Journal of Engineering Pedagogy (iJEP), 4(1), p. 49. https://dx.doi.org/10.3991/ijep.v4i1.3280.

Fikar, P., Güldenpfennig, F. and Ganhör, R. (2018) 'The Use(fulness) of Therapeutic Toys', in Proceedings of the 2018 on Designing Interactive Systems Conference 2018 - DIS '18. New York, New York, USA: ACM Press, pp. 289-300. https://dx.doi.org/10.1145/3196709.3196721.

Furlong, L. et al. (2018) 'Mobile apps for treatment of speech disorders in children : An evidence-based analysis of quality and efficacy', PLoS ONE, 13(8), pp. 1-12. doi: https://doi.org/10.1371/journal.pone.0201513.

Jadi, A. M. (2019) 'Improving the communication for children with speech disorders using the smart toys', arXiv, 10(3), pp. 25-40. https://dx.doi.org/10.5121/ijaia.2019.10303.

Kudrowitz, B. M. and Wallace, D. R. (2010) 'The play pyramid: a play classification and ideation tool for toy design', International Journal of Arts and Technology, 3(1), pp. 36-56. https://dx.doi.org/10.1504/IJART.2010.030492.

Maier, J. R. A. and Fadel, G. M. (2009) 'Affordance based design: A relational theory for design', Research in Engineering Design, 20(1), pp. 13-27. https://dx.doi.org/10.1007/s00163-008-0060-3.

Mertala, P. et al. (2016) 'The value of toys: 6-8-year-old children's toy preferences and the functional analysis of popular toys', International Journal of Play, 5(1), pp. 17-27. https://dx.doi.org/10.1080/21594937.2016.1147291.

Norman, D. A. (1999) ‘Affordance, conventions, and design', Interactions, 6(3), pp. 38-43. https://dx.doi.org/10.1145/301153.301168.

Pahl, G. et al. (2007) Engineering design: A systematic approach, Engineering Design: A Systematic Approach. https://dx.doi.org/10.1007/978-1-84628-319-2.

Rivera, D. et al. (2016) 'Smart Toys Designed for Detecting Developmental Delays', pp. 1-22. https://dx.doi.org/10.3390/s16111953.

Da Silva, J. R. S. et al. (2016) 'Using therapeutic toys to facilitate venipuncture procedure in preschool children', Pediatric Nursing, 42(2), pp. 61-68.

Sureeyatanapas, P. (2016) 'Comparison of rank-based weighting methods for multi-criteria decision making', Kku Engineering Journal, 43(S3), pp. 376-379. https://dx.doi.org/10.14456/kkuenj.2016.134.

Williams, S. E. and Matesi, D. V (1988) 'Therapeutic Intervention With an Adapted Toy', American Journal of Occupational Therapy, 42(10), pp. 673-676. https://dx.doi.org/10.5014/ajot.42.10.673. 